\title{
Theory of unstable laser modes: edge waves and fractality
}

\author{
Michael Berry ${ }^{\mathrm{a}, *}$, Cornelis Storm ${ }^{\mathrm{b}}$, Wim van Saarloos ${ }^{\mathrm{b}}$ \\ ${ }^{a}$ H.H. Wills Physics Laboratory, Tyndall Avenue, Bristol BS8 1TL, UK \\ b Instituut-Lorentz, Leiden University, P.O. Box 9506, 2300 RA Leiden, Netherlands \\ Received 15 June 2001; received in revised form 18 July 2001; accepted 22 July 2001
}

\begin{abstract}
For large Fresnel numbers $N$, unstable laser modes are highly irregular and resemble fractals. To explore this, we derive an explicit formula for the lowest-loss mode of a one-dimensional laser (i.e. where the cavity is two dimensional) in terms of edge-diffracted waves, and demonstrate its accuracy for large $N$. Between the size $a$ of the mirror (outer scale), and the inner scale $a / N$, there is no distinguished scale, and the graph of mode intensity has a fractal dimension close to 2. Near the inner scale, the scaling is scale dependent, and the crossover is described by an explicit formula for a 'local fractal dimension' $D(K)$, describing the mode on scales near $\Delta x=a /(2 \pi N K)$. As $K$ increases through the inner scale $K=1, D(K)$ decreases from 2 when $K \ll 1$ to 1 when $K \gg 1$ (reflecting the smoothness of the mode on fine scales). (C) 2001 Published by Elsevier Science B.V.
\end{abstract}

Keywords: Fractals; Unstable lasers; Edge waves; Asymptotics

\section{Introduction}

In a stable laser, where the cavity is bounded by concave mirrors, the intensity profile of the lowestloss mode, regarded as a function of a transverse coordinate $x$ perpendicular to the symmetry axis, is closely approximated by a narrow gaussian, and the edges of the mirrors play a negligible role [1]. If one of the mirrors is reversed (Fig. 1), the laser becomes unstable, and the mode spreads to fill the space between the mirrors, with energy leaking away beyond their edges. It was observed many years ago [2,3] that the profiles of these unstable modes are irregular, and more so as the wavelength $\lambda=2 \pi / k$ decreases.

\footnotetext{
${ }^{*}$ Corresponding author. Fax: +44-117-925-5624.
}

Recently, it was suggested $[4,5]$ that the repeated geometrical magnifications associated with the instability might imply that the modes have a fractal structure, and an attempt has been made [6] to calculate the fractal dimension $D$ of the intensity profile by numerical and analytical means. The self-similarity associated with the magnification has been studied in Ref. [7].

Our purpose here is to examine more carefully the scaling structure of the lowest-loss mode of an unstable laser, over the range from the 'outer scale' $\Delta x_{\text {outer }} \sim a$ down to the 'inner scale' $\Delta x_{\text {inner }} \sim \lambda L / a$, where $a$ is the size of the feedback (small) mirror and $L$ is the distance between the mirrors. On scales smaller than $\Delta x_{\text {inner }}$, the mode is a smooth function of $x$. As we show in Sections 3 and 4, the mode is indeed a fractal, in the usual sense that there is no distinguished scale between $\Delta x_{\text {outer }}$ and $\Delta x_{\text {inner. }}$. In Section 4 we show that successive 


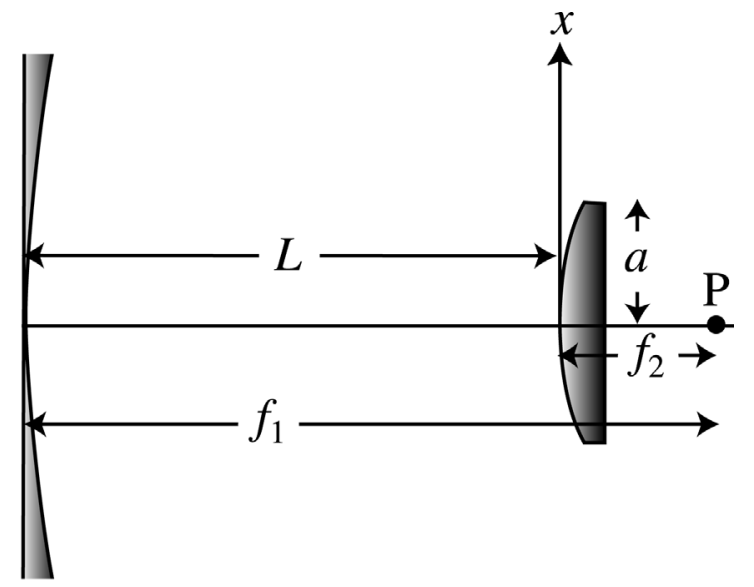

Fig. 1. A confocal unstable laser cavity. The two mirrors share a common focal point $\mathrm{P}$. We calculate mode profiles at the small mirror, that is for $|x|<a$.

magnifications, provided these do not reach down to $\Delta x_{\text {inner }}$, will indicate a dimension $D=2$. Near $\Delta x_{\text {inner }}$ however, the relation between different scales becomes nonuniform. It is possible to describe the crossover behaviour by defining a 'local fractal dimension', depending on wave number, and we calculate this crossover explicitly. (Moreover, the scaling appears to depend on position as well as scale.)

To obtain these results, we employ an explicit analytical expression for the mode (Section 2), valid in the asymptotic (short-wave) limit in which the inner and outer scales are well separated. This is a simplified version of a mathematical technique devised by Horwitz [2], or of its physical equivalent, namely the 'virtual-source' technique of Southwell [3]. This method reveals that the fine structure in the mode is a small-amplitude decoration, arising from edge waves, of the main beam (the main beam itself is geometrically propagated). There are two simplifications that enable us to get a usable formula. First, in the domain $|x|<a$ the edge waves can be represented trigonometrically, rather than in terms of Fresnel integrals. Second, the small- $\lambda$ asymptotic limit means that multiple edge diffraction can be neglected.

The scaling behaviour of unstable laser modes should be contrasted with that of the superficially similar wave of the Talbot effect. This is the overlap and interference of many edge-diffracted waves after diffraction by a Ronchi grating (alternating transparent and opaque bars with sharp edges); it was recently shown [8] that the transverse beam profile in almost all planes is a fractal, as here, but the graph of intensity has $D=3 / 2$ rather than $D=2$. The explanation given here, of the fractality of unstable laser modes, attests to the continuing fertility of Thomas Young's edge waves, almost exactly two centuries after he discovered them.

\section{The mode formula}

According to standard laser theory [1], each mode $u(x)$ is determined self-consistently by the requirement that after a round trip of the cavity (two reflections and two propagations) the wave returns to its original form, up to a constant multiplier $\gamma$ (the mode eigenvalue). For a strip resonator where the beam is limited by the width $2 a$ of the feedback mirror, assuming paraxial propagation and measuring $x$ in units of $a$, self-consistency leads to the integral equation

$\gamma u(x)=\sqrt{\frac{A}{2 \pi \mathrm{i} M}} \int_{-1}^{1} \mathrm{~d} y \exp \left\{\frac{1}{2} \mathrm{i} A\left(y-\frac{x}{M}\right)^{2}\right\} u(y)$,

where $M$ is the magnification; for an unstable laser, $M>1$. This equation applies to any unstable laser; for the particular case of a confocal laser (Fig. 1),

$M=f_{1} / f_{2} ; \quad A=\frac{2 \pi M^{2} a^{2}}{(M+1) \lambda L} \equiv \frac{4 \pi M^{2}}{\left(M^{2}-1\right)} N$,

in which the last equality defines the Fresnel number $N$. The integral operator in Eq. (1) is nonunitary, mathematically because of the finite integration limits $x= \pm 1$ and physically because of the loss of light at the edges of the mirror. Therefore $|\gamma|<1$.

We want to solve Eq. (1) in the asymptotic limit $A \gg 1$, in which scaling behaviour emerges (because the inner and outer scales are widely sepa- 
rated). In this paper we will concentrate on the lowest-loss mode, namely the solution $u(x)$ with the largest eigenvalue, for which $|\gamma|$ is closest to unity.

Numerically, there at least three options, and we have checked that they lead to identical results. First, the integral in Eq. (1) can be discretized, and the lowest-loss eigenvector of the resulting matrix determined by diagonalization. Second, $u(x)$ can be represented as a truncated Fourier series, again leading to a matrix diagonalization problem. Third Eq. (1) can be solved by iteration, starting from almost any initial function $u_{0}(x)$, for example $u_{0}(x)=1$ :

$u_{n+1}(x)=\sqrt{\frac{A}{2 \pi \mathrm{i} M}} \int_{-1}^{1} \mathrm{~d} y \exp \left\{\frac{1}{2} \mathrm{i} A\left(y-\frac{x}{M}\right)^{2}\right\} u_{n}(y)$,
$u_{0}=1$.

The first two methods are poor for large $A$. The third method converges quickly; it is the basis of the virtual-source technique [3], and also of the method we use here, following and simplifying [2].

When $A \gg 1$, the integral in Eq. (3) oscillates rapidly, and the principal contributions are localized at the single stationary-phase point $y=x / M$ (which always lies within the range of integration), and the two endpoints $x= \pm 1$. The stationaryphase contribution corresponds to geometrical optics, and dominates numerically. It is obtained by evaluating the gaussian integral that results from extending the integration range to infinity, giving

$u_{1}(x) \approx \frac{u_{0}(x / M)}{\sqrt{M}}=\frac{1}{\sqrt{M}}$,

whence the asymptotic eigenvalue is

$\gamma \rightarrow \frac{1}{\sqrt{M}} \quad$ as $A \rightarrow \infty$.

This unsurprising result describes the geometrical intensity loss factor $1 / M$ from rays that miss the feedback mirror after each round trip of the cavity, because of the magnification. The convergence onto Eq. (5) is accompanied by oscillations, as has been noted before [2]. In Appendix A we give a theory for the dominant Fourier component of these oscillatory corrections; Fig. 2 shows that

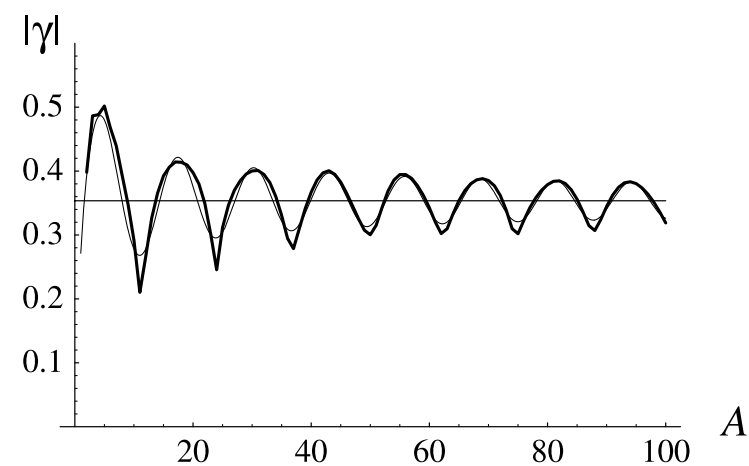

Fig. 2. Convergence of the largest eigenvalue $|\gamma|$ (thick curve) to $1 / \sqrt{M}$ (straight line) as $A$ increases, for $M=8$, together with the approximation (A.4) (thin curve).

when added to Eq. (5) this gives an accurate description of the eigenvalues, even for quite small $A$.

The endpoint contributions correspond to Young's edge waves; they can be approximated by local linearization of the phase of the integrand. For the first stage, this procedure gives (including the geometrical contribution)

$$
\begin{gathered}
u_{1}(x) \approx \frac{1}{\sqrt{M}}\left(1-\sqrt{\frac{\mathrm{i}}{2 \pi A}}\left(\frac{\exp \left\{\frac{1}{2} \mathrm{i} A(1-x / M)^{2}\right\}}{(1-x / M)}\right.\right. \\
\left.\left.+\frac{\exp \left\{\frac{1}{2} \mathrm{i} A(1+x / M)^{2}\right\}}{(1+x / M)}\right)\right)
\end{gathered}
$$

It is possible to extend Eqs. (4) and (6) by including higher-order terms in $1 / \sqrt{A}$ [2], but for present purposes the lowest-order theory is sufficient.

Now follow two crucial observations, that will greatly simplify further iterations. First, the singularities at $|x|=M$ lie outside the range $|x| \leqslant 1$ : the edges of the shadows are magnified, so they miss the mirror. This means that the edge waves are trigonometric functions, rather than the Fresnel integrals that result from the exact integration of Eq. (3). Second, the amplitudes of the edge waves are smaller by a factor $1 / \sqrt{A}$ than the ray contribution. This means that when incorporating Eq. (6) into the integration of Eq. (3) to get $u_{2}(x)$, $u_{3}(x)$, etc, it is sufficient to include only the stationary-point contributions when integrating the 
oscillatory terms: the endpoints will contribute quantities $1 / A$ and smaller, and so can be neglected in the asymptotic limit. Physically, this amounts to ignoring multiple edge diffraction, and treating the propagation of the edge waves, after their first creation, as purely geometric, that is, as edge rays.

The incorporation of these observations into the iteration of Eq. (3) is based on the following remarkable identity, describing the geometrical propagation and magnification of edge waves:

$$
\begin{aligned}
& \frac{1}{\sqrt{\alpha_{n}}} \int_{-1}^{1} \frac{\mathrm{d} y}{1-y / M^{n}} \exp \left\{\frac { 1 } { 2 } \mathrm { i } A \left[(y-x / M)^{2}\right.\right. \\
& \left.\left.+\left(1-y / M^{n}\right)^{2} \alpha_{n}\right]\right\} \\
& \approx \sqrt{\frac{2 \pi \mathrm{i}}{A \alpha_{n+1}}} \frac{\exp \left\{\frac{1}{2} \mathrm{i} A\left(1-y / M^{n+1}\right)^{2} \alpha_{n+1}\right\}}{1-x / M^{n+1}},
\end{aligned}
$$

where $\approx$ denotes the leading-order large- $A$ contribution, from the stationary-phase point, and

$\alpha_{n}=\frac{1}{\sum_{m=0}^{n-1} M^{-2 m}}=\frac{1-M^{-2}}{1-M^{-2 n}}$.

In conjunction with Eq. (3), this result gives the $n$th iteration of the initial wave as

$$
\begin{gathered}
u_{n}(x) \approx \frac{1}{M^{n / 2}}\left(1-\sqrt{\frac{\mathrm{i}}{2 \pi A}} \sum_{s=1}^{n} \frac{1}{\sqrt{\alpha_{s}}}\left[f\left(\frac{x}{M^{s}}, A \alpha_{s}\right)\right.\right. \\
\left.\left.+f\left(-\frac{x}{M^{s}}, A \alpha_{s}\right)\right]\right),
\end{gathered}
$$

where

$f(x, A) \equiv \frac{\exp \left\{\frac{1}{2} \mathrm{i} A(1-x)^{2}\right\}}{1-x}$.

Numerical explorations of this sum over edge waves show that when $n$ is not too large, Eqs. (8)(10) give results indistinguishable from those given by the exact iteration (3). However, when $n$ gets too large, the approximate and exact iterations diverge. Now we will explain this phenomenon, and show that it does not frustrate the application of Eq. (9) to approximate the mode.

Inspection of the terms in Eq. (9) shows that the term $s=1$ oscillates fastest. As $s$ increases, the terms oscillate more slowly, until if $n$ is large enough they eventually become essentially constant in the physical range $|x| \leqslant 1$. When does this happen? It is reasonable to define the terms as 'essentially constant' when the (dimensionless) wave number

$k(s)=\frac{\mathrm{d}(\text { phase })}{\mathrm{d} x} \approx \frac{A \alpha_{s}}{M^{s}}$ for large $s$,

which gets smaller as $s$ increases, reaches unity. This happens when

$s=s_{\max }=\frac{\log \left\{A\left(1-M^{-2}\right)\right\}}{\log M}$.

For $s>s_{\max }$, magnification has so stretched the edge waves that their diffraction oscillations are larger than the small mirror. Further iteration beyond $n=s_{\max }$ merely contaminates the constant term 1 in Eq. (9). If too many of these essentially constant edge wave terms are included (in fact a number of order $n-s_{\max } \sim \sqrt{A}$ ), their sum gets comparable with the original constant term 1 , and it is no longer justified to ignore the endpoint terms in their iterations. This is a kind of asymptotic resurgence: the high-order 'oscillatory' terms bite back and dominate the constant (i.e. lowestorder) term.

This failure of approximate iteration for large $n$ makes it natural to stop the iteration at $n=s_{\max }$, and regard the sum as the asymptotic eigenfunction. Including normalization to order $1 / \sqrt{A}$, we obtain the main result of this section:

$$
\begin{aligned}
u(x) \approx \frac{1}{\sqrt{2}} & \left(1-\sqrt{\frac{\mathrm{i}}{2 \pi A}} \sum_{s=1}^{s_{\max }} \frac{1}{\sqrt{\alpha_{s}}}\left[f\left(\frac{x}{M^{s}}, A \alpha_{s}\right)\right.\right. \\
& \left.\left.+f\left(-\frac{x}{M^{s}}, A \alpha_{s}\right)\right]\right) .
\end{aligned}
$$

This formula incorporates the geometrical magnification ('monitor-outside-a-monitor' effect [7]) and also the phase and diffraction effects essential to understanding the scaling.

The expectation is that Eq. (13) will get more accurate as $A$ increases, and it does. This is illustrated by Figs. 3 and 4, which show comparisons of Eq. (13) with eigenfunctions computed by diagonalization of the (discretized) matrix in Eq. (1) or exact numerical iteration of Eq. (3). Note that 

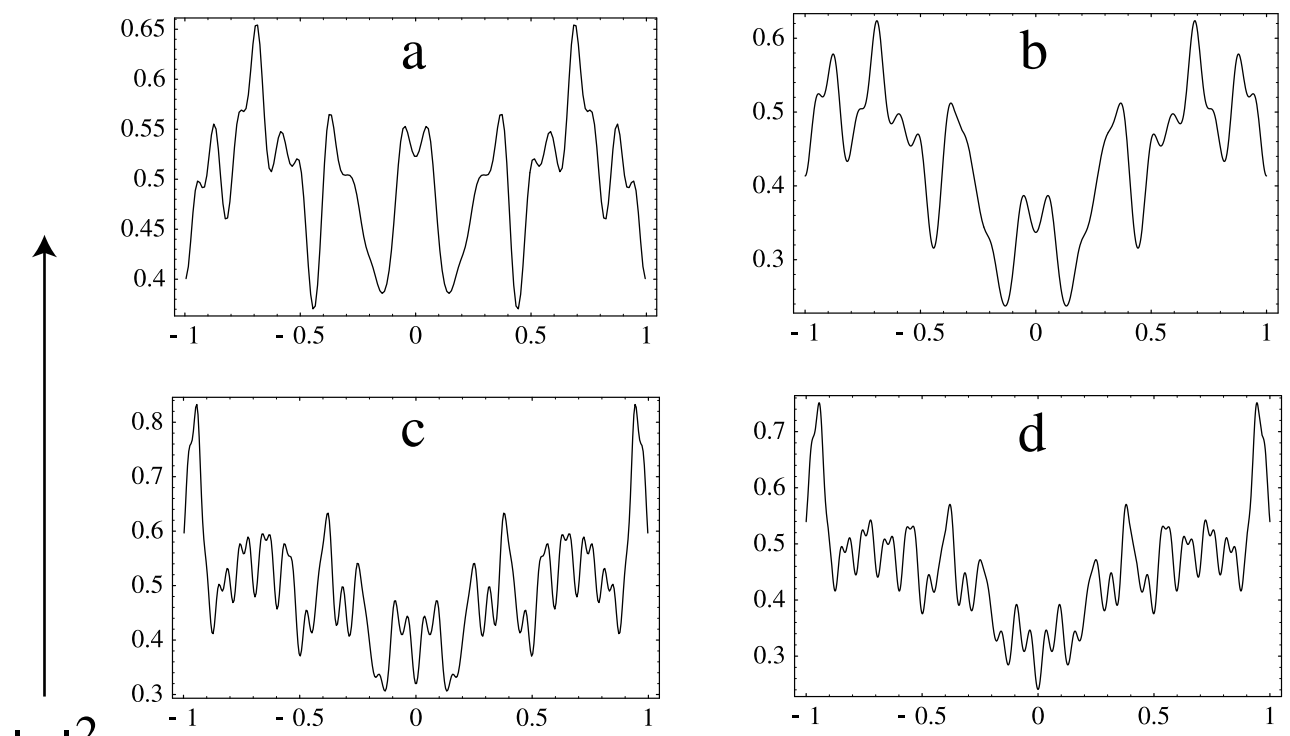

$|u|^{2}$
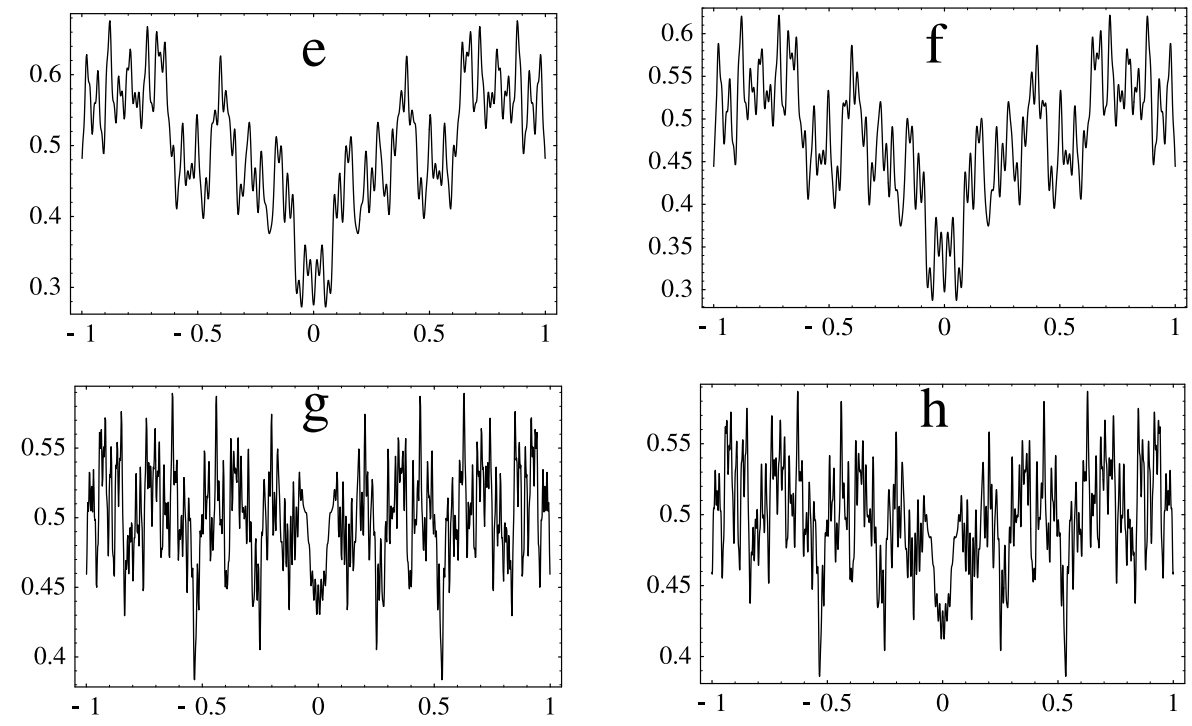

\section{$x$}

Fig. 3. (a,c,e,g): 'exact' mode intensities $|u(x)|^{2}$, computed by diagonalizing the integral Eq. (1) (a,c) or iteration from Eq. (3) (e,g). (b,d,f,h): mode intensities computed from the approximation (13). In (a,b), $A=100$; in (c,d), $A=200 ;$ in (e,f), $A=500$; in (g,h), $A=1000$. All calculations are for $M=2$.

to obtain the 'exact' eigenfunction for $A=1000$ by diagonalization requires at least a $2000 \times 2000$ matrix, whereas Eq. (13) includes only nine edge waves.

\section{Scaling of the edge wave sum}

Since the simple and explicit formula (13) is a very accurate representation of $u(x)$ when $A$ is 


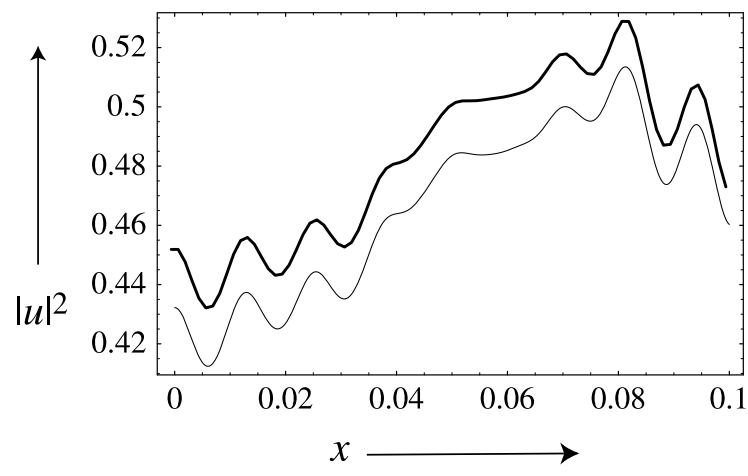

Fig. 4. Thick curve: magnification of Fig. 3g; (thin curve): magnification of Fig. 3 h. The curves have been displaced vertically for clarity.

large, we can use it to study the scaling behaviour of the mode. From Eq. (10), the exponent in each oscillatory term is a quadratic function of $x$, rather than a linear function. This gives rise to complicated substructure in the Fourier transform of $u(x)$, as has been noted already [6] and as we will discuss in Section 4. To side step this complexity, we now employ a more natural strategy, based on linearization of the exponent.

Locally, the (dimensionless) wave number corresponding to the term $s$ in the edge wave sum is

$$
\begin{aligned}
k(s ; x) & =\frac{\mathrm{d}(\text { phase })}{\mathrm{d} x}=\frac{A\left(1-x / M^{s}\right) \alpha_{s}}{M^{s}} \\
& =\frac{A\left(1-x / M^{s}\right)\left(1-M^{-2}\right)}{M^{s}-M^{-s}} .
\end{aligned}
$$

This will be a useful description of the local oscillations near $x$ if there are many such oscillations in small ranges of $x$, which is the case when $A$ is large. However, for small $x$, or for any $x$ when $M^{s} \gg 1$, we can replace Eq. (14) by the $x$-independent wave number

$k(s)=\frac{A\left(1-M^{-2}\right)}{M^{s}-M^{-s}}$.

It is convenient to work not with $k(s)$ but with the scaled wave number

$K(s) \equiv \frac{2 k(s)}{A\left(1-M^{-2}\right)}=\frac{2}{\left(M^{s}-M^{-s}\right)}$

and its inverse function
$s(K)=\frac{\log \left(1+\sqrt{1+K^{2}}\right)-\log K}{\log M}$.

The fastest oscillations $(s=1)$ correspond to $K=$ $2 /\left(M-M^{-1}\right)$, and the slowest oscillations $(k \sim 1$, i.e. $s \sim s_{\max }$ in Eq. (12)) correspond to $K \sim 2 / A$.

The wave numbers $K$ form a discrete set, because $s$ is an integer. However, in several circumstances we can regard the spectrum as continuous: under poor spectral resolution, when $s$ is large enough, for $M$ close to unity, or by averaging over a range of $A$ as we will describe later. Since the spacing in $s$ is uniform, we obtain the power spectrum from Eqs. (13) and (17) as

$P(K)=\frac{1}{\alpha_{s(K)}}\left|\frac{\mathrm{d} s(K)}{\mathrm{d} K}\right|$.

A short calculation shows that this is proportional to the universal (that is $M$-independent) function

$P(K)=\frac{1}{K\left(1+\sqrt{1+K^{2}}\right) \sqrt{1+K^{2}}}$.

If $u(x)$ were a fractal function with dimension $D$, that is if the graph of $\operatorname{Re} u, \operatorname{Im} u$, and also $|u|^{2}$ (see Ref. [9]) were fractal curves, then $P(K)$ would be the power law

$P_{\text {fractal }}(K)=\frac{1}{K^{5-2 D}}$

(for derivations of this result, see Refs. [10-12]). But $P(K)$ is not a pure power law, so that the mode does not possess the uniform scaling (between the inner and outer scales) that would correspond to a pure fractal. Instead, the spectrum (19) varies from $1 / K$ for small $K$, that is for the coarsest scales - corresponding to $D=2$ - to $1 / K^{3}$ for large $K$, that is for the finest scales, where the mode is smooth-corresponding to $D=1$.

This suggests defining a scale-dependent fractal dimension, inspired by Eq. (20), namely

$D(K) \equiv \frac{1}{2}\left(5+\frac{\mathrm{d} \log P(K)}{\mathrm{d} \log K}\right)$,

for which a short calculation gives

$D(K)=1+\frac{1}{2}\left(\frac{1}{1+K^{2}}+\frac{1}{\sqrt{1+K^{2}}}\right)$. 


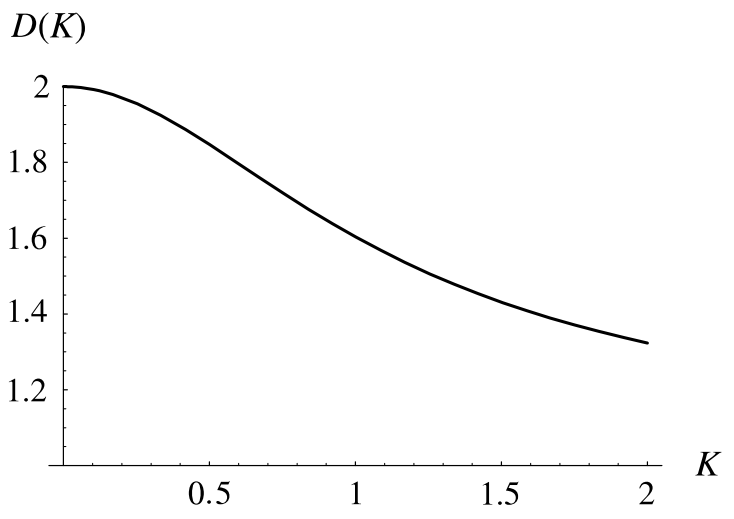

Fig. 5. The scale-dependent dimension $D(K)$ (Eq. (22)) characterizing unstable laser modes.

This formula is our main result. For an unstable laser with Fresnel number $N, D(K)$ describes scaling of the lowest-loss mode over scales near $\Delta x=a /(2 \pi N K)$. Fig. 5 shows the form of $D(K)$, decreasing smoothly from 2 to 1 .

We should remark that there is some flexibility in the attribution of a fractal dimension to a spectrum that is not a power law. Instead of Eq. (20), we could study the variance $V(X)=\langle(u(x+$ $\left.X)-u(x))^{2}\right\rangle$, where the average is over $x$; for a fractal, this scales as $V(X) \sim X^{4-2 D}$ [11]. Calculating $V$ from the spectrum (19), and determining a local dimension $D_{V}(X)$ by the analogue of Eq. (21), leads to $D_{V}(X=2 \pi / K)$ different from Eq. (22), though with similar qualitative behaviour.

On scales well between the outer and inner extremes, the fractal dimension is $D=2$. However, attempts to measure the fractal dimension by numerical techniques that sample $u(x)$ on successively smaller scales would eventually be frustrated by the nonconstancy of $D(K)$, which gets smaller as the crossover is probed. The dimension finally reached by such techniques, corresponding to the finest scales $K(s=1)=2 /\left(M-M^{-1}\right)$ (cf. Eq. (16)), would be

$D_{\min }(M) \equiv D\left(\frac{2}{M-M^{-1}}\right)=1+\frac{M^{2}\left(M^{2}-1\right)}{\left(M^{2}+1\right)^{2}}$

and is illustrated in Fig. 6. $D_{\min }$ increases from 1 when $M=1$ and approaches 2 as $M \rightarrow \infty$. For

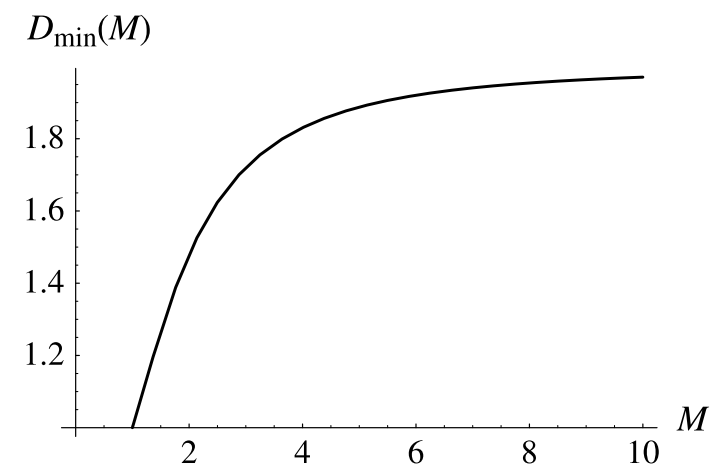

Fig. 6. Dimension $D_{\min }(M)$ (Eq. (23)) corresponding to the finest scales of the mode for magnification $M$.

$M=2$, for example, $D_{\min }=37 / 25=1.48 \ldots$, close to a value observed before [4].

\section{Fractal implications}

Now we return to the Fourier transform of $u(x)$, or rather its absolute square, namely the power spectrum $P(k)$ (note that we here revert to the original Fourier variable $k$, rather than the scaled $K)$. The upper curve in Fig. 7 shows that for a

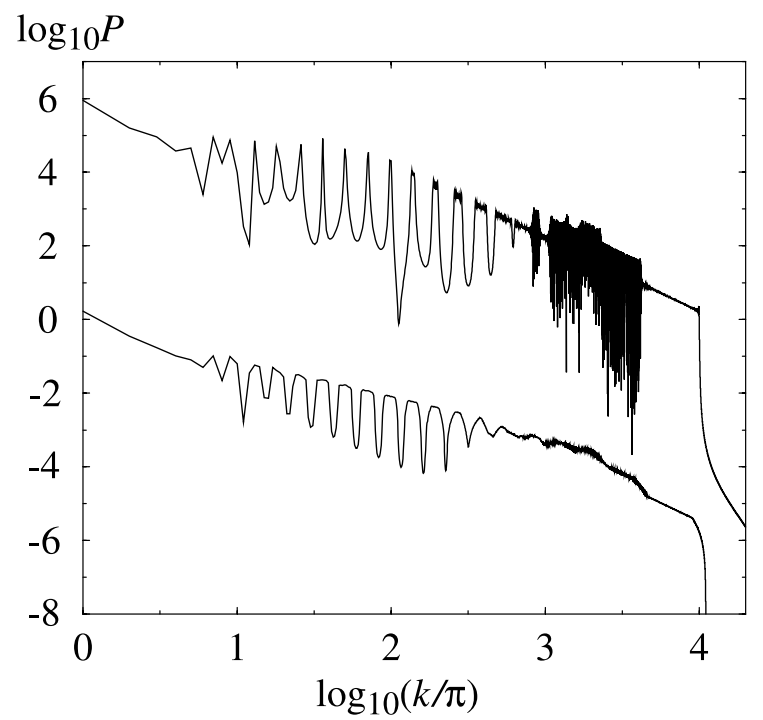

Fig. 7. Upper curve: spectrum $P(k)$ for the lowest-loss mode with $N=1000, M=1.4$ (i.e. $A=25656.3, s_{\max }=28$ ). Lower curve: the spectrum averaged over 100 equally spaced $N$ values between 900 and 1100. (For clarity, the two curves have been displaced vertically.) 
given mode $P(k)$ possesses a complicated structure. Some of this has been interpreted [6] in terms of the individual magnified Fresnel edge-diffraction patterns; the edges of the 'bands' can be calculated from Eq. (14) by setting $x= \pm 1$. The slope -2 of the straight-line parts of the spectrum was also noted [6], and interpreted according to Eq. (20) as implying $D=3 / 2$ for $u(x)$, in contradiction to the variable scaling described in the previous section.

However, the slope -2 is an artifact, arising from the extrapolation, to the whole pattern, of the Fourier structure of the individual edge waves comprising it. To clarify this, we suppress the individual edge wave contributions by averaging over a range of $A$. As the lower curve in Fig. 7 shows, this smoothing all but destroys the slope -2 . Nevertheless, considerable structure remains. To get smoother spectra, we could average over a larger range of values of $A$, or increase $A$. Here we adopt the latter strategy, choosing a value of $M$ closer to 1 (cf. Eq. (2)), thereby incorporating more edge waves (cf. Eq. (12)) and increasing the overlap of their corresponding Fourier bands.

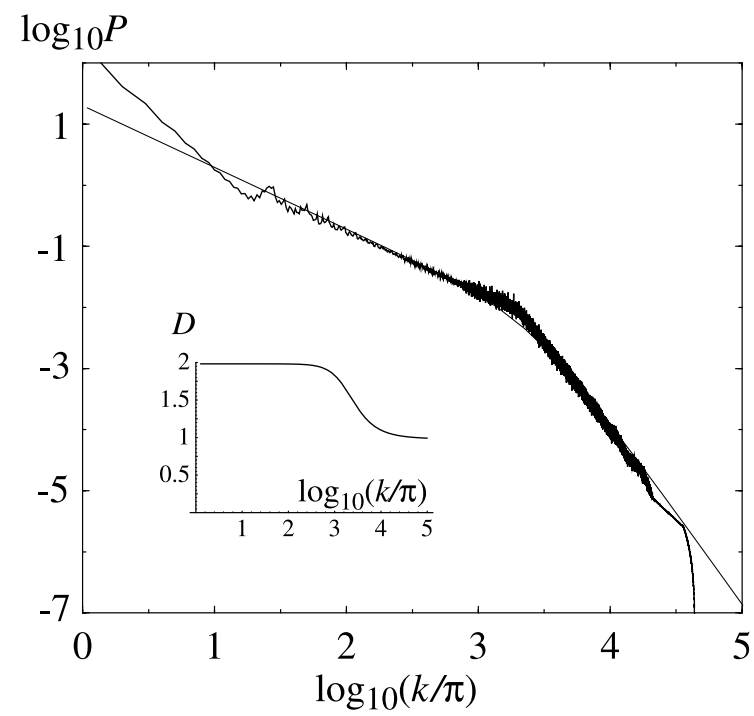

Fig. 8. Jagged curve: spectrum $P(k)$ for $M=1.1$, averaged over 50 equally spaced $N$ values between 900 and 1100 (for $\left.N=1000, A=72406.2, s_{\max }=99\right)$. Smooth curve: theoretical spectrum (19), with $K(k)$ given by Eq. (16). Inset: local fractal dimension $D$ (Eq. (22)), plotted similarly.
As Fig. 8 shows, this results in a much smoother power spectrum. In the range between the inner and outer scales, the curve shows a crossover between two segments with clearly defined slopes -1 and -3 , corresponding to the small and large $K$ limits of the universal scaling spectral function $P(K)$ (Eq. (19)). This averaged power spectrum is closely fitted with no adjustable parameters by $P(K)$ when plotted in terms of $\log k$ rather than $K$.

The crossover occurs near $K=1$, that is $k=$ $2 N / a$. The change of slope appears fairly sharp, in apparent contrast to Fig. 5, showing smooth variation of the local exponent of the power spectrum-equivalent to the local dimension $D(K)$. This raises an important point. When $D$ is plotted as a function of $\log K$ (inset of Fig. 8) the crossover region is compressed, and two extremes are emphasized. The large- $k$ extreme, that is $D=$ 1 , merely reflects the ultimate smoothness of $u(x)$ below the inner scale. But the small- $k$ behaviour, that is $D=2$, is significant: probing the mode, by successive magnification revealing scales smaller than the mirror but still much larger than the inner scale, will indicate an almost constant fractal dimension $D=2$. However, as the crossover wave number $k=2 N / a$ is approached the measured dimension will change, as described in the previous section (Eq. (22)).

\section{Concluding remarks}

The scale-dependent scaling behaviour revealed by our analysis is an asymptotically emergent phenomenon: it requires large $A$, yet is essentially determined by corrections to the geometricaloptics limit. The full scaling behaviour is subtle and complicated and would be more complicated still if we had included the weak dependence on $x$ (cf. the remarks after Eq. (14)). In a way this phenomenon evokes the length of England's coastline, that contributed to the discovery of fractals [13]: on ever-finer scales, the length increases. For coastlines, the length changes between scales according to a fixed law, over a very large range. By contrast, in unstable laser modes the scaling between scales is not exactly constant. Although many physical systems exhibit fractal scaling only 
over a limited range of length scales, the unstable laser provides a rare example where the crossover can be calculated explicitly.

Our scaling predictions can be tested experimentally. The simplest procedure would be as follows. (1) Measure the mode intensities $|u(x)|^{2}$ for an ensemble of unstable lasers with a range of parameters near given values of $N$ and $M$. (2) For each laser, calculate the power spectrum of $|u(x)|^{2}$ (which should have similar fractal structure to the power spectrum of $u(x)$ itself). (3) Average these power spectra over the ensemble of lasers. (4) Compare a $\log -\log$ plot of the averaged power spectrum with the predictions of Eq. (19), and identify the limiting fractal dimensions 2 and 1 (as illustrated in Section 4), and the crossover behaviour near $k=2 N / a$. We anticipate that for very large $A$-greatly exceeding those in our simulations - the predicted scaling behaviour will be apparent in individual modes, without the need for averaging over an ensemble.

Scale-dependent scaling near the inner scale should not be confused with multifractality [14]. This is much wilder behaviour, where regions with different fractal dimensions are themselves fractally distributed. It would be interesting to explore the possibility that unstable laser modes have multifractal aspects, but we have not done so.

Nevertheless, the scaling behaviour of unstable lasers is more subtle and complicated than in superficially similar situations. We have already mentioned the apparent resemblance between unstable laser modes and the waves in the Talbot effect, but in the Talbot effect the scaling is uniform (albeit interestingly spatially anisotropic [8]), so the wave is genuinely fractal. Mathematically, the mode formula (13) looks similar to the Weierstrass-Mandelbrot function [11]. Both involve repeated magnifications. However, in the Weierstrass-Mandelbrot case the oscillatory terms are trigonometric exponentials, so the function is a Fourier series, and the scaling is uniform, whereas for laser modes the phases of the oscillatory terms are quadratic, and the coefficients $\alpha_{s}$ destroy the uniform scaling.

For the lowest-loss modes $u(x, y)$ of unstable lasers with two-dimensional mirrors, the oscillatory structure at each point $(x, y)$ can still be rep- resented as a finite sum of waves, associated with edge rays originating from points where lines through $(x, y)$ intersect the edges and their images perpendicularly, and this sum can be used to determine the scaling behaviour. The theory has interesting geometrical aspects, and will be published separately.

\section{Acknowledgements}

Michael Berry is grateful for the hospitality of the Instituut-Lorentz, Leiden, where this work started. We thank Professor J.P. Woerdman for bringing this problem to our attention, and for useful discussions.

\section{Appendix A. Oscillatory behaviour of the eigen- value}

In terms of the iterates $u_{n}(x)$, defined by Eq. (3), the eigenvalue is determined by the limit

$\gamma=\lim _{n \rightarrow \infty} \frac{u_{n+1}(x)}{u_{n}(x)}$.

For large $A$ we can use the asymptotic approximation (9). The only difference between $u_{n}(x)$ and $u_{n+1}(x)$, apart from the extra factor $1 / \sqrt{M}$ responsible for the leading-order behaviour (5) of the eigenvalue, is an additional term in the edgewave sum, with index $s=n+1$. Using Eqs. (8) and (10), we have

$$
\begin{aligned}
& \frac{1}{\sqrt{\alpha_{n}}}\left[f\left(\frac{x}{M^{n}}, A \alpha_{n}\right)+f\left(-\frac{x}{M^{n}}, A \alpha_{n}\right)\right] \\
& \rightarrow \frac{2}{\sqrt{1-M^{-2}}} \exp \left\{\frac{1}{2} \mathrm{i} A\left(1-M^{-2}\right)\right\} \quad \text { as } n \rightarrow \infty .
\end{aligned}
$$

Thus Eq. (A.1) gives

$$
\begin{aligned}
\gamma= & \frac{1}{\sqrt{M}}\left[1-\sqrt{\frac{2 \mathrm{i}}{\pi A\left(1-M^{-2}\right)}} \exp \left\{\frac{1}{2} \mathrm{i} A\left(1-M^{-2}\right)\right\}\right] \\
& +\mathrm{O}\left(A^{-1}\right)
\end{aligned}
$$


and hence, for the modulus

$$
\begin{aligned}
|\gamma|= & \frac{1}{\sqrt{M}}\left[1-\sqrt{\frac{2}{\pi A\left(1-M^{-2}\right)}}\right. \\
& \left.\times \cos \left\{\frac{1}{2} A\left(1-M^{-2}\right)+\frac{1}{4} \pi\right\}\right]+\mathrm{O}\left(A^{-1}\right) .
\end{aligned}
$$

\section{References}

[1] A.E. Siegman, Lasers, University Science Books, Mill Valley, 1986.

[2] P. Horwitz, Asymptotic theory of unstable resonator modes, J. Opt. Soc. Am. 63 (1973) 1528-1543.

[3] W.H. Southwell, Unstable-resonator-mode derivation using virtual-source theory, J. Opt. Soc. Am. 3 (1986) 1885-1891.

[4] G.P. Karman, J.P. Woerdman, Fractal structure of eigenmodes of unstable-cavity lasers, Opt. Lett. 23 (1998) 19091911.
[5] G.P. Karman, G.S. McDonald, G.H.C. New, J.P. Woerdman, Fractal modes in unstable resonators, Nature 402 (1999) 138.

[6] G.H.C. New, M.A. Yates, J.P. Woerdman, G.S. McDonald, Diffractive origin of fractal resonator modes, Opt. Commun. 193 (2001) 261-266.

[7] J. Courtial, M.J. Padgett, Monitor-outside-a-monitor effect and self-similar fractal structure in the eigenmodes of unstable optical resonators, Phys. Rev. Lett. 85 (2000) 5320-5323.

[8] M.V. Berry, S. Klein, Integer, fractional and fractal Talbot effects, J. Mod. Opt. 43 (1996) 2139-2164.

[9] M.V. Berry, Quantum fractals in boxes, J. Phys. A 26 (1996) 6617-6629.

[10] S. Orey, Gaussian sample functions and the Hausdorff dimension of level crossings, Z. Wahrscheinlichkeitstheorie 15 (1970) 249-256.

[11] M.V. Berry, Z.V. Lewis, On the Weierstrass-Mandelbrot fractal function, Proc. Roy. Soc. A 370 (1980) 459-484.

[12] K. Falconer, Fractal Geometry: Mathematical Foundations and Applications, Wiley, New York, 1990.

[13] B.B. Mandelbrot, The Fractal Geometry of Nature, Freeman, San Francisco, 1982.

[14] B.B. Mandelbrot, Multifractals and $1 / f$ Noise, Springer, New York, 1998. 